\title{
A Copula-Based Index to Measure Directional Reflection Asymme- try for Trivariate Copulas
}

\author{
Ali Dolati ${ }^{1}$ and Ahmad Alikhani-Vafa ${ }^{1}$ \\ ${ }^{1}$ Department of Statistics, College of Mathematics, Yazd University, 89195-741, Yazd, Iran.
}

Received: 21/09/2018, Revision received: 28/03/2019, Published online: 29/08/2019

\begin{abstract}
We propose a copula-based index to detect the reflection asymmetry in trivariate distributions. The proposed index is based on the definition of directional reflection asymmetry over the set of directions. We derive the asymptotic distribution of the rank-based estimator of the proposed index. The value of the index and the direction in which the asymmetry occurs are easily computed, and we illustrate it with a simulation study and a real data analysis.
\end{abstract}

Keywords. Asymptotic Normality, Copula, Empirical Processes, Reflection Asymmetry, Test of Asymmetry.

MSC: 62H05; 62E10.

\section{Introduction}

Suitable models are often needed for multivariate data in many applications when departure from normality assumption can be found in data. Following Sklar (1959) the joint cumulative distribution function $H$ of the vector $\mathbf{X}=\left(X_{1}, \ldots, X_{d}\right)$, with the continuous marginal distribution functions $F_{i}, i=1, \ldots, d$, can then be expressed as $H\left(x_{1}, \ldots, x_{d}\right)=C\left\{F_{1}\left(x_{1}\right), \ldots, F_{d}\left(x_{d}\right)\right\}$, in terms of a unique copula $C:[0,1]^{d} \rightarrow[0,1]$, which is itself the joint distribution function of the vector $\mathbf{U}=\left(U_{1}, \ldots, U_{d}\right)=\left(F_{1}\left(X_{1}\right), \ldots, F_{d}\left(X_{d}\right)\right)$ of uniform $(0,1)$ random variables. Modeling multidimensional data using copulas has found many applications in the last two decades; see Nelsen (2006), Trivedi and Zimmer (2007), Cherubini et al. (2011) and Joe (2015) for a review and references therein. The main reason for the interest in copulas is that they allow for the modelling of different dependence structures of data. A natural question is how does one choose an appropriate copula for a given data set? One model selection approach is to consider the goodness-of-fit tests for copulas; see Genest et al. (2009) and Berg (2009). A complimentary to the goodness-of-fit methods are first testing the copula structure of interest

Corresponding Author: Ali Dolati (adolati@yazd.ac.ir) 
such as symmetries to suggest a sensible class of parametric families of copulas.

Fitting an inappropriate model to the data by neglecting some properties such as the asymmetries can lead to incorrect conclusions. Even though there is a unique way of defining symmetry about a point in one-dimension, there exist several definitions for symmetry in the multidimensional case. Nelsen (1993) discusses several definitions for bivariate symmetry. A kind of symmetry that exhibited, for instance, by elliptically contoured distributions, is the radial symmetry or reflection symmetry which is also known as central or diagonal symmetry (Sefling (2006)).

Throughout this paper, we assume that the center of symmetry is known. Hence, without loss of generality, we consider the problem of reflection about zero. Formally, the random vector $\mathbf{X}$ is reflection symmetric if $\mathbf{X}={ }^{D}-\mathbf{X}$, where $={ }^{D}$ denotes the equality in distribution. Equivalently, $\mathbf{X}$ is reflection symmetric if, and only if, $H(\mathbf{x})=\bar{H}(-\mathbf{x})$, for all $\mathbf{x}=\left(x_{1}, . ., x_{d}\right) \in \mathbb{R}^{d}$, where $\bar{H}(\mathbf{x})=P\left(X_{1}>x_{1}, \ldots, X_{d}>x_{d}\right)$ is the survival function associated with $H$. Consequently, reflection symmetric random variables $X_{1}, \ldots, X_{d}$ must be marginally symmetric, i.e., $F_{i}(x)=$ $1-F_{i}(-x), i=1, \ldots, d$, for all $x \in \mathbb{R}$. Of course, the converse is false. Many commonly used distributions such as multivariate normal and the other elliptically contoured distributions are reflection symmetric (Fang et al. (1989)). Let $\widehat{C}$ denote the survival copula associated with the copula $C$, defined by $\widehat{C}\left(u_{1}, \ldots, u_{d}\right)=\bar{C}\left(1-u_{1}, \ldots, 1-u_{d}\right)$ for all $\left(u_{1}, \ldots, u_{d}\right) \in[0,1]^{d}$, which is the joint distribution of the vector $\left(1-U_{1}, \ldots, 1-U_{d}\right)$ of uniform $(0,1)$ random variables and $\bar{C}\left(u_{1}, \ldots, u_{d}\right)=P\left(U_{1}>u_{1}, \ldots, U_{d}>u_{d}\right)$ is the survival function associated with the copula $C$. For $d=2, \widehat{C}\left(u_{1}, u_{2}\right)=u_{1}+u_{2}-1+C\left(1-u_{1}, 1-u_{2}\right)$ and the expression for $\widehat{C}$ becomes more complicated for $d>2$. If $F_{i} \mathrm{~s}, i=1, \ldots, d$ are symmetric about zero, then the distribution of the vector $\left(U_{1}, \ldots, U_{d}\right)=\left(F_{1}\left(X_{1}\right), \ldots, F_{d}\left(X_{d}\right)\right)$ is symmetric about the point $(1 / 2, \ldots ., 1 / 2)$ if, and only if, $C\left(u_{1}, \ldots, u_{d}\right)=\widehat{C}\left(u_{1}, \ldots, u_{d}\right)$, for all $\left(u_{1}, \ldots, u_{d}\right) \in[0,1]^{d}$. Thus the problem of investigating the symmetry of a bivariate distribution can be reduced to that of investigating the symmetry of its associated copula as far as we know the center of symmetry.

A natural approach to measure the amount of asymmetry in a copula is based on a suitable distance between the copula and its associated survival copula. For the case $d=2$, recently several copula-based measures of reflection asymmetry and statistical tests of symmetry are defined and studied in literature; see Buzebda and Cherfi (2012), Dehgani et al. (2013), Genest and Neślehová (2014), and Alikhani-Vafa and Dolati (2018). Rosco and Joe (2013) studied reflection asymmetry measures using an approach based on the univariate skewness to account the direction of the asymmetry. Modarres (2017) considered the problem of estimation of a bivariate reflection symmetric distribution. In the multivariate case, the situation is more complicated. Due to the complexity of the relation between the copula and its associated survival copula, the extension of the distance-based bivariate asymmetry measures beyond dimension 2 is a difficult task. Recently, Krupskii (2017) proposed a multivariate measure of reflection asymmetry for copulas based on a weighting function.

As we will see in Section 2, this index measures the strength of reflection asymmetry, but it could not account for the correct direction of reflection asymmetry in dimensions $d>2$. To solve this problem, we propose a copula-based index to measure the strength and also the direction of reflection asymmetry in trivariate case. The index is based on the definition of directional reflection asymmetry over the set of directions. The rest of the paper is organized as follows. In Section 2 we propose the new index. In Section 3 we investigate the asymptotic normality of the empirical counterpart of the proposed index. The power of the test of sym- 
metry based on the proposed index and that of Krupskii (2017) is compared in Section 4. The measure is then applied to analyze a real data set in Section 4 . Section 5 concludes the paper with a discussion.

\section{A directional Reflection Asymmetry Measure}

Let $\left(U_{1}, \ldots U_{d}\right)$ be a vector of uniform $(0,1)$ random variables having a copula $C$. Krupskii (2017) proposed a measure of multivariate reflection asymmetry of the form

$$
G\left(\Delta_{R}, k\right)=\int_{[0,1]^{d}} w\left(u_{1}, \ldots, u_{d}\right)\left(C\left(u_{1}, \ldots, u_{d}\right)-\widehat{C}\left(u_{1}, \ldots, u_{d}\right)\right) d u_{1} \ldots d u_{d}
$$

where $w\left(u_{1}, \ldots, u_{d}\right)=\left(\frac{1}{2}-\frac{1}{d} \sum_{i=1}^{d} u_{i}\right)^{k} I_{\left\{\sum_{i=1}^{d} u_{i} \leq \frac{d}{2}\right\}}$ is a weighting function and $k$ is a positive integer. A closed expression for this index is given by

$$
G\left(\Delta_{R} ; k\right)=\frac{(-d)^{d}}{(k+1)(k+2) \ldots(k+d)} E\left[\left|\frac{1}{2}-\frac{1}{d} \sum_{j=1}^{d} U_{j}\right|^{k+d} . \operatorname{sign}\left(\frac{1}{2}-\frac{1}{d} \sum_{j=1}^{d} U_{j}\right)\right]
$$

For a given copula $C$, let $G(C)=G\left(\Delta_{R} ; k\right) / m_{d}$ where the normalizing constant $m_{d}$ is the maximal value of $G\left(\Delta_{R} ; k\right)$. The index $G(C)$ ranges over $(-1,1)$, with larger values indicating more reflection asymmetry in the copula $C$. As the following example shows this index fails to detect the reflection asymmetry in many situations.

Example 2.1. Let $\left(U_{1}, U_{2}, U_{3}\right)$ follow the trivariate Clayton copula (see; Nelsen (2006)) given by

$$
C_{1}\left(u_{1}, u_{2}, u_{3}\right)=\left(u_{1}^{-\theta}+u_{2}^{-\theta}+u_{3}^{-\theta}-2\right)^{-1 / \theta}, \quad \theta>0
$$

Consider the rotated Clayton copula associated with the vector $\left(U_{1}, U_{2}, 1-U_{3}\right)$, which is given by $C_{2}\left(u_{1}, u_{2}, u_{3}\right)=P\left(U_{1} \leq u_{3}, U_{2} \leq u_{2}, 1-U_{3} \leq u_{3}\right)=C_{1}\left(u_{1}, u_{2}, 1\right)-C_{1}\left(u_{1}, u_{2}, 1-u_{3}\right)$. Both $C_{1}$ and $C_{2}$ are reflection asymmetric, i.e., $C_{1} \neq \widehat{C}_{1}$ and $C_{2} \neq \widehat{C}_{2}$, but for a common level of dependency, say, Kendall's $\tau=0.5$, we see that $G\left(C_{1}\right)=0.5112$ and $G\left(C_{2}\right)=0.0006 \cong 0$. Thus the index $G\left(\right.$.) fails to measure the asymmetry of the copula $C_{2}$.

In the following, we propose a generalization of (2.1) to resolve this problem. First, we define the directional reflection asymmetric.

Definition 2.1. For $\alpha_{i} \in\{-1,1\}, i=1, \ldots, d$, we say that the vector $\left(U_{1}, \ldots, U_{d}\right)$ of uniform $(0,1)$ random variables is reflection asymmetric in the direction $\left(\alpha_{1}, \ldots, \alpha_{d}\right)$ if

$$
\left(\alpha_{1}\left(U_{1}-\frac{1}{2}\right), \ldots, \alpha_{d}\left(U_{d}-\frac{1}{2}\right)\right) \neq^{D}\left(\alpha_{1}\left(\frac{1}{2}-U_{1}\right), \ldots, \alpha_{d}\left(\frac{1}{2}-U_{d}\right)\right) .
$$


For the directions $\left(\alpha_{1}, \ldots, \alpha_{d}\right)=(1, \ldots, 1)$ and $\left(\alpha_{1}, \ldots, \alpha_{d}\right)=(-1, \ldots,-1)$, the condition $(2.3)$ is equivalent to $C\left(u_{1}, \ldots, u_{d}\right) \neq \widehat{C}\left(u_{1}, \ldots, u_{d}\right)$, for some $\left(u_{1}, \ldots, u_{d}\right) \in[0,1]^{d}$, where $C$ is the copula associated with the vector $\left(U_{1}, \ldots, U_{d}\right)$. For a reflection symmetric copula, we have

$$
\sum_{j=1}^{d} \alpha_{j}\left(U_{j}-\frac{1}{2}\right)={ }^{D} \sum_{j=1}^{d} \alpha_{j}\left(\frac{1}{2}-U_{j}\right),
$$

or equivalently

$$
\sum_{j=1}^{d} \alpha_{j} U_{j}-\frac{1}{2} \sum_{j=1}^{d} \alpha_{j}={ }^{D} \frac{1}{2} \sum_{j=1}^{d} \alpha_{j}-\sum_{j=1}^{d} \alpha_{j} U_{j}
$$

Let

$$
T=\frac{1}{2 d} \sum_{j=1}^{d} \alpha_{j}-\frac{1}{d} \sum_{j=1}^{d} \alpha_{j} U_{j} .
$$

For a reflection symmetric copula, we have $T={ }^{D}-T$; that is $T$ is symmetric about zero. Thus, any univariate measure of skewness for $T$ would be an index for measuring the amount of asymmetry for a vector $\left(U_{1}, \ldots, U_{d}\right)$ or its associated copula $C$, in the direction $\left(\alpha_{1}, \ldots, \alpha_{d}\right)$. A natural index of asymmetry could be

$$
\lambda^{\left(\alpha_{1}, \ldots, \alpha_{d}\right)}=\frac{E\left[|T|^{k} \cdot \operatorname{sgn}(T)\right]}{m(k, d)},
$$

where $k$ is a positive integer, $d \geq 2$ is the dimension of the copula $C$ and $m(k, d)$ is a normalizing constant to ensure that the index falls in the interval $(-1,1)$. This index measures the amount and direction of reflection asymmetry that exist in the copula $C$. It is proportional to the index $G\left(\Delta_{R} ; k\right)$ defined by $(2.1)$ when $\left(\alpha_{1}, \ldots, \alpha_{d}\right)=(1, \ldots, 1)$. If $C\left(u_{1}, \ldots, u_{d}\right) \neq \widehat{C}\left(u_{1}, \ldots, u_{d}\right)$, for some $\left(u_{1}, \ldots, u_{d}\right) \in[0,1]^{d}$, then this reflection asymmetry could be observed in some direction.

In the multivariate case, the situation is more complicated and we consider just the trivariate case. In this case, the direction $\left(\alpha_{1}, \alpha_{2}, \alpha_{3}\right)$ of asymmetry belongs to one of the following sets

$$
D_{1}=\{(1,1,1),(1,1,-1),(1,-1,1),(1,-1,-1)\}
$$

or

$$
D_{2}=\{(-1,-1,-1),(-1,-1,1),(-1,1,-1),(-1,1,1)\}
$$

Note that $D_{1}$ and $D_{2}$ are two complementary sets. In view of (2.3), for instance, the directions $(1,1,1)$ and $(-1,-1,-1)$ are the same. We consider $D_{1}$ as the set of directions of asymmetry. For simplicity, we use the following notions in the sequel

$$
\lambda_{1}=\lambda^{(1,1,1)}, \quad \lambda_{2}=\lambda^{(1,1,-1)}, \quad \lambda_{3}=\lambda^{(1,-1,1)} \quad \text { and } \quad \lambda_{4}=\lambda^{(1,-1,-1)}
$$

Definition 2.2. Let $\left(X_{1}, X_{2}, X_{3}\right)$ be a random vector with the associated copula $C$ and let $\lambda^{\left(\alpha_{1}, \alpha_{2}, \alpha_{2}\right)}$ denote the index defined by (2.4). Let $\left(\alpha_{1}^{*}, \alpha_{2}^{*}, \alpha_{3}^{*}\right)$ be the direction for which the absolute value of the index has a maximum value. We define $\lambda^{\left(\alpha_{1}^{*}, \alpha_{2}^{*}, \alpha_{3}^{*}\right)}$ as the index of directional asymmetry of the copula $C$ in the direction $\left(\alpha_{1}^{*}, \alpha_{2}^{*}, \alpha_{3}^{*}\right)$. 
We consider the normalizing constant $m(k, 3)$ in $(2.4)$ as the value of the index for a vector $\left(U_{1}^{*}, U_{2}^{*}, U_{3}^{*}\right)$ with the maximum reflection asymmetry in some direction $\left(\alpha_{1}, \alpha_{2}, \alpha_{3}\right)$. For this purpose, we use the family of singular copulas that considered in Krupskii (2017). Let $U_{1}$, $U_{2}$ and $U_{3}$ be three independent uniform $(0,1)$ random variables. For $0 \leq \theta \leq 1$, consider the vector $\left(U_{1}^{*}, U_{2}^{*}, U_{3}^{*}\right)=\left(U_{1}, U_{1}, U_{1}\right)$ if $U_{1}<\theta$ and $\left(U_{1}^{*}, U_{2}^{*}, U_{3}^{*}\right)=\left(U_{1}, U_{2}, U_{3}\right)$ if $U_{1}>\theta$. So, we have

$$
E\left[|T|^{k} \cdot \operatorname{sgn}(T)\right]= \begin{cases}\int_{0}^{\theta}\left(\frac{1}{2}-u\right)^{k} d u+\int_{0}^{1} \int_{0}^{1} \int_{\theta}^{1}|T|^{k} \cdot \operatorname{sgn}(T) d u_{1} d u_{2} d u_{3}, & \theta<\frac{1}{2} \\ \int_{0}^{\frac{1}{2}}\left(\frac{1}{2}-u\right)^{k} d u-\int_{\frac{1}{2}}^{\theta}\left(u-\frac{1}{2}\right)^{k}+\int_{0}^{1} \int_{0}^{1} \int_{\theta}^{1}|T|^{k} \cdot \operatorname{sgn}(T) d u_{1} d u_{2} d u_{3}, & \theta>\frac{1}{2} .\end{cases}
$$

We maximize the numerator of (2.4) for the copula of the vector $\left(U_{1}^{*}, U_{2}^{*}, U_{3}^{*}\right)$. After some algebra the derivative of $E\left[|T|^{k} \cdot \operatorname{sgn}(T)\right]$ with respect to $\theta$ is given by

$$
\frac{\partial}{\partial \theta} E\left[|T|^{k} \cdot \operatorname{sgn}(T)\right]=\left|\frac{1}{2}-\theta\right|^{k} \cdot \operatorname{sgn}\left(\frac{1}{2}-\theta\right)-\frac{\left(\frac{3}{2}-\theta\right)^{k+2}-2\left|\frac{1}{2}-\theta\right|^{k+2} \cdot \operatorname{sgn}\left(\frac{1}{2}-\theta\right)-\left(\frac{1}{2}+\theta\right)^{k+2}}{3^{k}(k+1)(k+2)} .
$$

This is a polynomial of order $k+2$ and the roots can be obtained numerically for each $k$. For instance, when $k=2$ we obtain that the maximum is attained at $\theta=0.655$ and $m(k, 3)=0.02957$. When $k=3$ the maximum is attained at $\theta=0.67$ and $m(k, 3)=0.01264$. For choosing a suitable value for $k$, we have examined the values of $\lambda_{i}, i=1,2,3,4$ for $1 \leq k \leq 80$ as a function of $k$.

Figure 1 shows the values of $\lambda_{1}$ as a function of $k$ for Clayton and Gumbel copulas. The results were similar for $\lambda_{i}, i=2,3,4$ and the other reflection asymmetric copulas. As we see from Figure 1 small values of $k$ leads to this observation that the measures $\lambda_{i}$ are less sensitive to an asymmetric dependence and large values of $k$ result in a large variability of $\lambda_{i}$. So selecting $4 \leq k \leq 10$ can be a rational choice.

We also computed and plotted the power of the corresponding asymmetry test statistic as a function of $k$ for several reflection asymmetric copulas in Figure 2 in Section 4 , it can be seen that the corresponding statistical test with $\lambda_{1}$ has the largest power for $5 \leq k \leq 8$. So we have selected the value $k=7$ in our calculations. In this section, the normalizing constant $m(k, 3)$ is given by 0.00046 . The following example shows the value of the $\lambda_{i}, i=1,2,3,4$ for several reflection asymmetric copulas.

Example 2.2. Consider the trivariate Gumbel family of copulas (see; Nelsen (2006)) given by

$$
G_{1}\left(u_{1}, u_{2}, u_{3}\right)=\exp \left[\left(-\log \left(u_{1}\right)\right)^{\theta}+\left(-\log \left(u_{2}\right)\right)^{\theta}+\left(-\log \left(u_{3}\right)\right)^{\theta}\right]^{1 / \theta}, \quad \theta \in[1, \infty) .
$$

Let $G_{2}, G_{3}$, and $G_{4}$ be the rotated versions of $G_{1}$; i.e, the copulas associated with the vectors $\left(U_{1}, U_{2}, 1-U_{3}\right),\left(U_{1}, 1-U_{2}, U_{3}\right)$ and $\left(U_{1}, 1-U_{2}, 1-U_{3}\right)$, respectively. Let $C_{2}, C_{3}$ and $C_{4}$ be the rotated versions of the trivariate Clayton copula (2.2). The values of the directional asymmetry measures $\lambda_{i}, i=1,2,3,4$ are given in Table 1 . As we note, while the copulas $C_{2}, C_{3}, C_{4}, G_{2}, G_{3}$, and $G_{4}$ are reflection asymmetric, the value of the index $\lambda_{1}$ is near zero for these copulas. The column $\left(\alpha_{1}^{*}, \alpha_{2}^{*}, \alpha_{3}^{*}\right)$ shows the direction of the reflection asymmetry for each copula. 

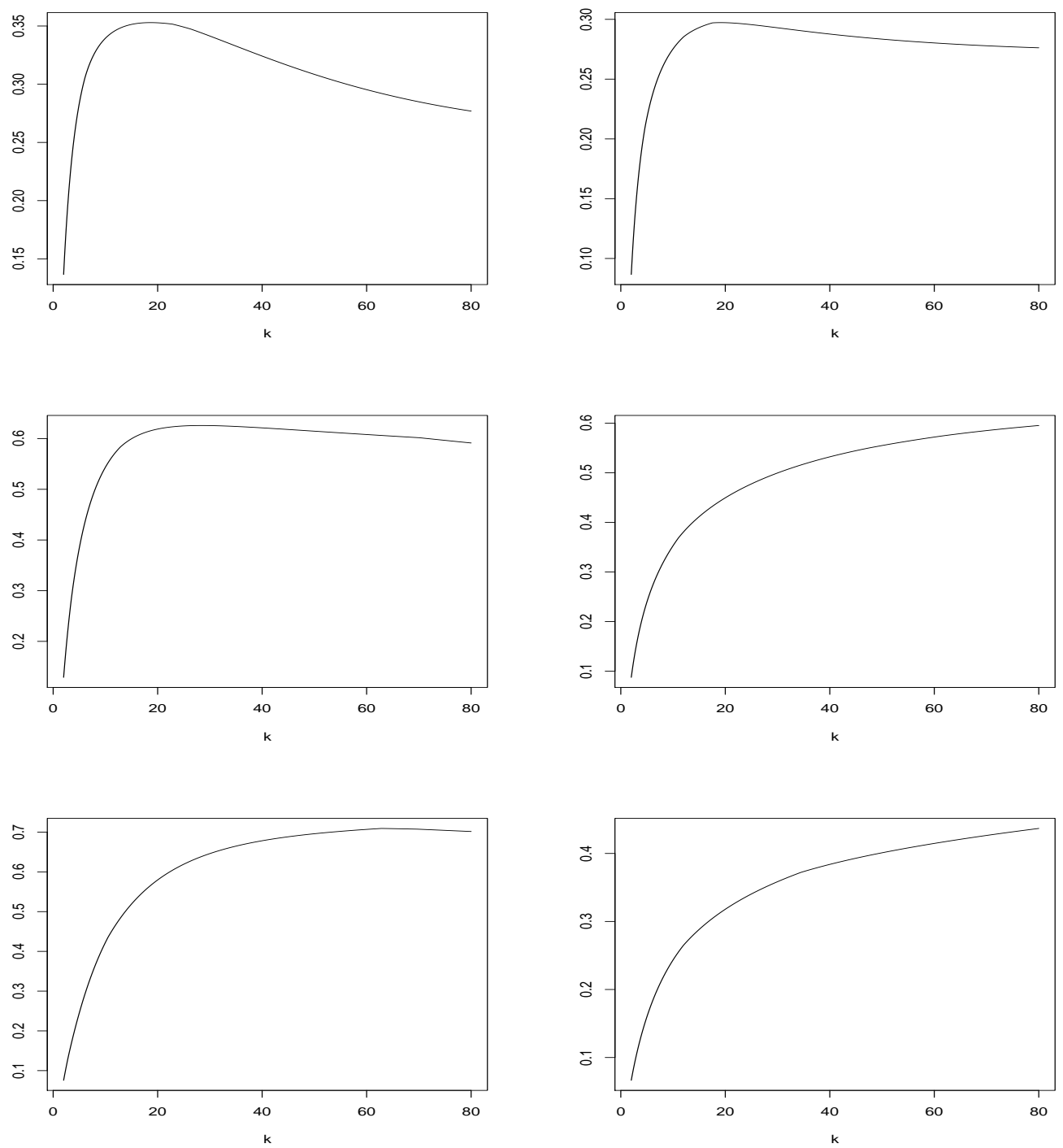

Figure 1: The plot of $\lambda_{1}$ as a function of $k$ for the Clayton copula (the left panels) and the Gumbel copula (the right panels) for Kendall's $\tau \in\{0.25,0.5,0.75\}$ (the top to the bottom ). 
Table 1: Values of $\lambda_{i}, i=1,2,3,4$, for the Clayton copula $\left(C_{1}\right)$ and its rotated versions $\left(C_{2}, C_{3}, C_{4}\right)$ and the Gumbel copula $\left(G_{1}\right)$ and its rotated versions $\left(G_{2}, G_{3}, G_{4}\right)$ for different values of Kendall's $\tau$.

\begin{tabular}{ccccccc}
\hline \hline Copula & $\tau$ & $\lambda_{1}$ & $\lambda_{2}$ & $\lambda_{3}$ & $\lambda_{4}$ & $\left(\alpha_{1}^{*}, \alpha_{2}^{*}, \alpha_{3}^{*}\right)$ \\
\hline \hline$C_{1}$ & 0.2 & $\mathbf{0 . 2 4 1}$ & 0.015 & 0.015 & 0.015 & $(1,1,1)$ \\
$C_{2}$ & 0.4 & 0.004 & $\mathbf{0 . 4 6 6}$ & 0.004 & 0.005 & $(1,1,-1)$ \\
$C_{3}$ & 0.6 & -0.001 & 0.001 & $\mathbf{0 . 4 9 9}$ & -0.001 & $(1,-1,1)$ \\
$C_{4}$ & 0.8 & 0.000 & 0.000 & 0.000 & $\mathbf{0 . 3 0 2}$ & $(1,-1,-1)$ \\
\hline$G_{1}$ & 0.2 & $\mathbf{- 0 . 2 3 5}$ & 0.027 & 0.027 & -0.026 & $(1,1,1)$ \\
$G_{2}$ & 0.4 & 0.021 & $\mathbf{- 0 . 3 2 9}$ & -0.021 & 0.021 & $(1,1,-1)$ \\
$G_{3}$ & 0.6 & 0.007 & -0.007 & $\mathbf{- 0 . 2 6 9}$ & 0.007 & $(1,-1,1)$ \\
$G_{4}$ & 0.8 & 0.000 & 0.000 & 0.000 & $\mathbf{- 0 . 1 0 8}$ & $(1,-1,-1)$ \\
\hline
\end{tabular}

The value of the index $\lambda_{i}, i=1,2,3,4$, for the trivariate case could not be expressed in terms of the bivariate marginal indices. These indices discusses in the following. In the bivariate case we have two directions of radial asymmetry with directions $D_{1}=\{(1,1),(1,-1)\}$ or $D_{2}=\{(-1,-1),(-1,1\}$ and the index $(2.4)$ reduces to

$$
\lambda^{\left(\alpha_{1}, \alpha_{2}\right)}=\frac{E\left[|T|^{k} \cdot \operatorname{sgn}(T)\right]}{m(k, 2)} .
$$

Note that $\lambda^{(1,1)}$ and $\lambda^{(1,-1)}$ are the reflection asymmetry measures studied in Krupskii (2017) and Rosco and Joe (2013). The normalizing constant $m(k, 2)$ is the maximum reflection asymmetry obtained in Rosco and Joe (2013). Similar to the trivariate case we consider the set $D_{1}$ as the set of directions of asymmetry and for simplicity we use the following notions in the sequel

$$
\xi_{1}=\lambda^{(1,1)}, \quad \xi_{2}=\lambda^{(1,-1)}, \quad .
$$

Table 2 shows the values of the indices $\xi_{1}$ and $\xi_{2}$ for the bivariate marginal of rotated Clayton copula (denoted by $C_{3,12}, C_{3,13}, C_{3,23}$ ) and that of Gumbel copula (denoted by $G_{2,12}, G_{2,13}, G_{2,23}$ ) in Example 2.2.

Table 2: Values of $\xi_{1}$ and $\xi_{2}$ for the bivariate marginal of rotated Clayton copula (denoted by $C_{3,12}$, $C_{3,13}, C_{3,23}$ ) and that of Gumbel copula (denoted by $G_{2,12}, G_{2,13}, G_{2,23}$ ) in Example 2.2.

\begin{tabular}{cccc}
\hline \hline Copula & $\xi_{1}$ & $\xi_{2}$ & $\left(\alpha_{1}^{*}, \alpha_{2}^{*}\right)$ \\
\hline \hline$C_{3,12}$ & -0.0001 & 0.3790 & $(1,-1)$ \\
$C_{3,13}$ & 0.3803 & 0.0000 & $(1,1)$ \\
$C_{3,23}$ & -0.0002 & -0.3805 & $(1,-1)$ \\
\hline$G_{2,12}$ & -0.2074 & 0.0000 & $(1,1)$ \\
$G_{2,13}$ & 0.0007 & -0.2043 & $(1,-1)$ \\
$G_{2,23}$ & 0.0004 & -0.2061 & $(1,-1)$ \\
\hline
\end{tabular}




\section{Empirical Version and Asymptotic Normality}

Let $\left(X_{1 j}, X_{2 j}, X_{3 j}\right), j=1,2, \ldots, n$ be a sample of size $n$ from a vector $\left(X_{1}, X_{2}, X_{3}\right)$ of continuous random variables with the copula $C$. For $j=1, \ldots, n$, let $\left(R_{1 j}, R_{2 j}, R_{3 j}\right)$ denote the corresponding vectors of ranks, i.e., $R_{i j}=\sum_{l=1}^{n} \mathbb{I}\left\{X_{i l} \leq X_{i j}\right\}$, for $i=1,2,3$ and $1 \leq j \leq n$, where $\mathbb{I}\{A\}$ denotes the indicator function of the set $A$. Let

$$
C_{n}\left(u_{1}, u_{2}, u_{3}\right)=\frac{1}{n} \sum_{j=1}^{n} \mathbb{I}\left\{U_{1 j} \leq u_{1}, U_{2 j} \leq u_{2}, U_{3 j} \leq u_{3}\right\},
$$

with the pseudo-observations $U_{i j}=R_{i j} /(n+1), i=1,2,3, j=1,2, \ldots, n$, be the empirical copula (Fermanian et al. (2004)). Let

$$
T(u, v, w)=\frac{\alpha_{1}+\alpha_{2}+\alpha_{3}}{6}-\frac{\alpha_{1} u+\alpha_{2} v+\alpha_{3} w}{3},
$$

and

$$
B_{j}=T\left(\frac{R_{1 j}}{n+1}, \frac{R_{2 j}}{n+1}, \frac{R_{3 j}}{n+1}\right) .
$$

A plug-in rank-based estimator of the index $\lambda^{\left(\alpha_{1}, \alpha_{2}, \alpha_{3}\right)}$ defined by (2.4), is given by

$$
\begin{aligned}
\lambda_{n}^{\left(\alpha_{1}, \alpha_{2}, \alpha_{3}\right)} & =\frac{1}{m(k, 3)} \int_{[0,1]^{3}}\left|T\left(u_{1}, u_{2}, u_{3}\right)\right|^{k} \operatorname{sgn}\left(T\left(u_{1}, u_{2}, u_{3}\right)\right) d C_{n}\left(u_{1}, u_{2}, u_{3}\right) \\
& =\frac{1}{n \times m(k, 3)} \sum_{j=1}^{n}\left|B_{j}\right|^{k} \operatorname{sgn}\left(B_{j}\right) .
\end{aligned}
$$

The following theorem provides the asymptotic distribution of $\lambda_{n}^{\left(\alpha_{1}, \alpha_{2}, \alpha_{3}\right)}$.

Theorem 3.1. The asymptotic distribution of the empirical measure $\lambda_{n}^{\left(\alpha_{1}, \alpha_{2}, \alpha_{3}\right)}$ defined by (3.2) is given by

$$
\sqrt{n}\left(\lambda_{n}^{\left(\alpha_{1}, \alpha_{2}, \alpha_{3}\right)}-\lambda^{\left(\alpha_{1}, \alpha_{2}, \alpha_{3}\right)}\right) \longrightarrow^{w} Z_{\lambda}
$$

where $Z_{\lambda} \sim N\left(0, \sigma_{\lambda}^{2}\right)$ with

$$
\begin{aligned}
\sigma_{\lambda}^{2} & =\operatorname{var}\left(g\left(U_{1}, U_{2}, U_{3}\right)+\sum_{i=1}^{3} T_{i}(g)\left(U_{i}\right)\right) \\
g\left(u_{1}, u_{2}, u_{3}\right) & =\frac{1}{m(k, 3)}\left|\frac{1}{6} \sum_{i=1}^{3} \alpha_{i}-\frac{1}{3} \sum_{i=1}^{3} \alpha_{i} u_{i}\right|^{k} \operatorname{sgn}\left(\frac{1}{6} \sum_{i=1}^{3} \alpha_{i}-\frac{1}{3} \sum_{i=1}^{3} \alpha_{i} u_{i}\right), \\
T_{i}(g)\left(u_{i}\right) & =\int_{[0,1]^{3}} \dot{g}_{i}(x, y, z) I_{\left\{u_{i} \leq x\right\}} d C(x, y, z),
\end{aligned}
$$

where for $i=1,2,3, \dot{g}_{i}\left(u_{1}, u_{2}, u_{3}\right)=\frac{\partial}{\partial u_{i}} g\left(u_{1}, u_{2}, u_{3}\right)$. 
Proof. Let $D_{n}=\sqrt{n}\left(C_{n}-C\right)$ denote the empirical copula process (Fermanian et al. (2004)). Then we have

$$
\sqrt{n}\left(\lambda_{n}^{\left(\alpha_{1}, \alpha_{2}, \alpha_{3}\right)}-\lambda^{\left(\alpha_{1}, \alpha_{2}, \alpha_{3}\right)}\right)=\sqrt{n} \int_{[0,1]^{3}} g\left(u_{1}, u_{2}, u_{3}\right) d D_{n} .
$$

In view of Theorem 7 in Radulovic et al. (2017), we have

$$
\int_{[0,1]^{3}} g\left(u_{1}, u_{2}, u_{3}\right) d D_{n}=\int_{[0,1]^{3}}\left(g\left(u_{1}, u_{2}, u_{3}\right)+\sum_{i=1}^{3} T_{i}(g)\left(u_{i}\right)\right) d \alpha_{n}\left(u_{1}, u_{2}, u_{3}\right)+o_{p}(1),
$$

where

$$
\alpha_{n}\left(u_{1}, u_{2}, u_{3}\right)=\frac{1}{\sqrt{n}}\left(\sum_{j=1}^{n} \mathbb{I}\left\{U_{1 j} \leq u_{1}, U_{2 j} \leq u_{2}, U_{3 j} \leq u_{3}\right\}-C\left(u_{1}, u_{2}, u_{3}\right)\right),
$$

with the pseudo-observations $U_{i j}=R_{i j} /(n+1), i=1,2,3, j=1,2, \ldots, n$. If we take

$$
h\left(u_{1}, u_{2}, u_{3}\right)=g\left(u_{1}, u_{2}, u_{3}\right)+\sum_{i=1}^{3} T_{i}(g)\left(u_{i}\right)
$$

then the integral on the right-hand side of (3.3) is asymptotically equal to

$$
\frac{1}{\sqrt{n}} \sum_{i=1}^{n}\left\{h\left(U_{1 i}, U_{2 i}, U_{3 i}\right)-E\left[h\left(U_{1 i}, U_{2 i}, U_{3 i}\right)\right]\right\}
$$

where $\left(U_{1 i}, U_{2 i}, U_{3 i}\right), i=1, \ldots, n$ is a random sample of size $n$ from the vector $\left(U_{1}, U_{2}, U_{3}\right)$ with the copula $C$. An application of the central limit theorem yields the desired result.

Making inference based on Theorem 3.1 requires estimation of the asymptotic variance. We propose to estimate $\sigma_{\lambda}^{2}$ by the empirical variance of the (observable) sample $Z_{1}, \ldots, Z_{n}$, where $Z_{i}=h\left(U_{1 i}, U_{2 i}, U_{3 i}\right), i=1, \ldots, n$, as defined in (3.4).

Note that in applications, we first estimate the value of the proposed index for the data set, in four directions. Then, for the direction $\left(\alpha_{1}^{*}, \alpha_{2}^{*}, \alpha_{3}^{*}\right)$ with the largest value of the index, the test $H_{0}: \lambda^{\left(\alpha_{1}^{*}, \alpha_{2}^{*}, \alpha_{3}^{*}\right)}=0$ is performed. We can reject $H_{0}$ if $\left|\sqrt{n} \lambda_{n}^{\left(\alpha_{1}^{*}, \alpha_{2}^{*}, \alpha_{3}^{*}\right)}\right| \geq \hat{\sigma_{\lambda}} Z_{1-\frac{\alpha}{2}}$ where

$Z_{1-\frac{\alpha}{2}}$ is $1-\frac{\alpha}{2}$ quantile of the standard normal distribution. If the test was significant, then an appropriate copula will be selected via a copula goodness of fit test (see, e.g., Genest et al. (2009) and Berg (2009)) among the trivariate copulas that are asymmetric in that direction.

\section{Simulations and Applications}

\subsection{Simulations}

In this subsection, we study the power of the test statistics based on the sample versions of $\lambda_{1}$ and $\xi_{1}$. We compare the power of the test statistics for the asymptotic method obtained in 
Theorem 3.1 and the bootstrap or jackknife method applied in Krupskii (2017). We simulate from the bivariate and trivariate Clayton copula having a strong dependence in the lower tail and the Gumbel copula having a strong dependence in the upper tail. We consider two different sample sizes $n=100,200$ and five different degrees of dependence in terms of the Kendall's $\tau \in\{0.1,0.3,0.5,0.7,0.9\}$. The results are shown in Table 3 . The numbers in the first row of the table represent the power of the test statistics for asymptotic method obtained in Theorem 3.1 and the numbers in the second row show the power of the test statistics for the method applied in Krupskii (2017). All tests were carried out at the 5\% nominal level. As we see the power of the asymmetry test for the asymptotic method obtained in Theorem 3.1 is better than the bootstrap or jackknife method applied in Krupskii (2017) in almost all cases, unless when $\tau$ tends to 0.9 . Note that when $\tau$ tends to one the copula $C$ tends to the reflection symmetric copula $M$ (the Frechét-Hoeffding upper bound copula), thus this phenomenon is not wondering. We also plot the power of the test for the Clayton and the Gumbel copula for $1 \leq k \leq 20, n=200, d=3$ and Kendall's $\tau \in\{0.25,0.5,0.75\}$ in Figure 2. The maximum power obtained; for $4 \leq k \leq 8$. Therefore, selecting $k=7$ is a good choice. Results are similar in the bivariate case and for other reflection asymmetry copulas.

Table 3: Power of the reflection asymmetry test based on sample versions of the measures $\lambda_{1}$ (for dimension $d=3$ ) and $\xi_{1}$ (for dimension $d=2$ ) using 1000 random samples of size $n=100,200$ from the Clayton and the Gumbel copulas, with Kendall's $\tau \in\{0.1,0.3,0.5,0.7,0.9\}$. The numbers in the first row of the table show the power of the test by using the asymptotic method given in Theorem 3.1 and the numbers in the second row show the power of the test for the method applied in Krupskii (2017).

\begin{tabular}{|c|c|c|c|c|c|c|c|}
\hline \multirow{2}{*}{$\begin{array}{c}\text { Copula } \\
\text { Dimension }\end{array}$} & \multirow[b]{2}{*}{$\tau$} & \multicolumn{2}{|c|}{ Clayton } & \multicolumn{2}{|c|}{ Gumbel } & \multicolumn{2}{|c|}{ normal } \\
\hline & & $n=100$ & $n=200$ & $n=100$ & $n=200$ & $n=100$ & $n=200$ \\
\hline \multirow{10}{*}{$d=2$} & \multirow{2}{*}{0.1} & 0.199 & 0.252 & 0.096 & 0.152 & 0.520 & 0.500 \\
\hline & & 0.154 & 0.234 & 0.092 & 0.150 & 0.480 & 0.490 \\
\hline & \multirow{2}{*}{0.3} & 0.680 & 0.922 & 0.272 & 0.481 & 0.060 & 0.045 \\
\hline & & 0.631 & 0.916 & 0.261 & 0.476 & 0.057 & 0.040 \\
\hline & \multirow{2}{*}{0.5} & 0.933 & 1.000 & 0.367 & 0.632 & 0.510 & 0.037 \\
\hline & & 0.924 & 1.000 & 0.342 & 0.615 & 0.044 & 0.035 \\
\hline & \multirow{2}{*}{0.7} & 0.972 & 1.000 & 0.357 & 0.689 & 0.026 & 0.030 \\
\hline & & 0.984 & 1.000 & 0.319 & 0.671 & 0.019 & 0.029 \\
\hline & \multirow{2}{*}{0.9} & 0.444 & 1.000 & 0.007 & 0.266 & 0.002 & 0.003 \\
\hline & & 0.845 & 1.000 & 0.086 & 0.586 & 0.007 & 0.007 \\
\hline \multirow{10}{*}{$d=3$} & \multirow{2}{*}{0.1} & 0.219 & 0.358 & 0.162 & 0.387 & 0.055 & 0.051 \\
\hline & & 0.136 & 0.309 & 0.169 & 0.384 & 0.051 & .0 .045 \\
\hline & \multirow{2}{*}{0.3} & 0.802 & 0.972 & 0.569 & 0.864 & 0.065 & 0.046 \\
\hline & & 0.740 & 0.961 & 0.565 & 0.851 & 0.062 & 0.041 \\
\hline & \multirow{2}{*}{0.5} & 0.983 & 1.000 & 0.654 & 0.926 & 0.051 & 0.048 \\
\hline & & 0.975 & 1.000 & 0.630 & 0.903 & 0.046 & 0.045 \\
\hline & \multirow{2}{*}{0.7} & 0.998 & 1.000 & 0.593 & 0.908 & 0.027 & 0.036 \\
\hline & & 0.998 & 1.000 & 0.575 & 0.905 & 0.029 & 0.035 \\
\hline & \multirow{2}{*}{0.9} & 0.854 & 1.000 & 0.022 & 0.568 & 0.001 & 0.004 \\
\hline & & 0.992 & 1.000 & 0.227 & 0.805 & 0.009 & 0.017 \\
\hline
\end{tabular}



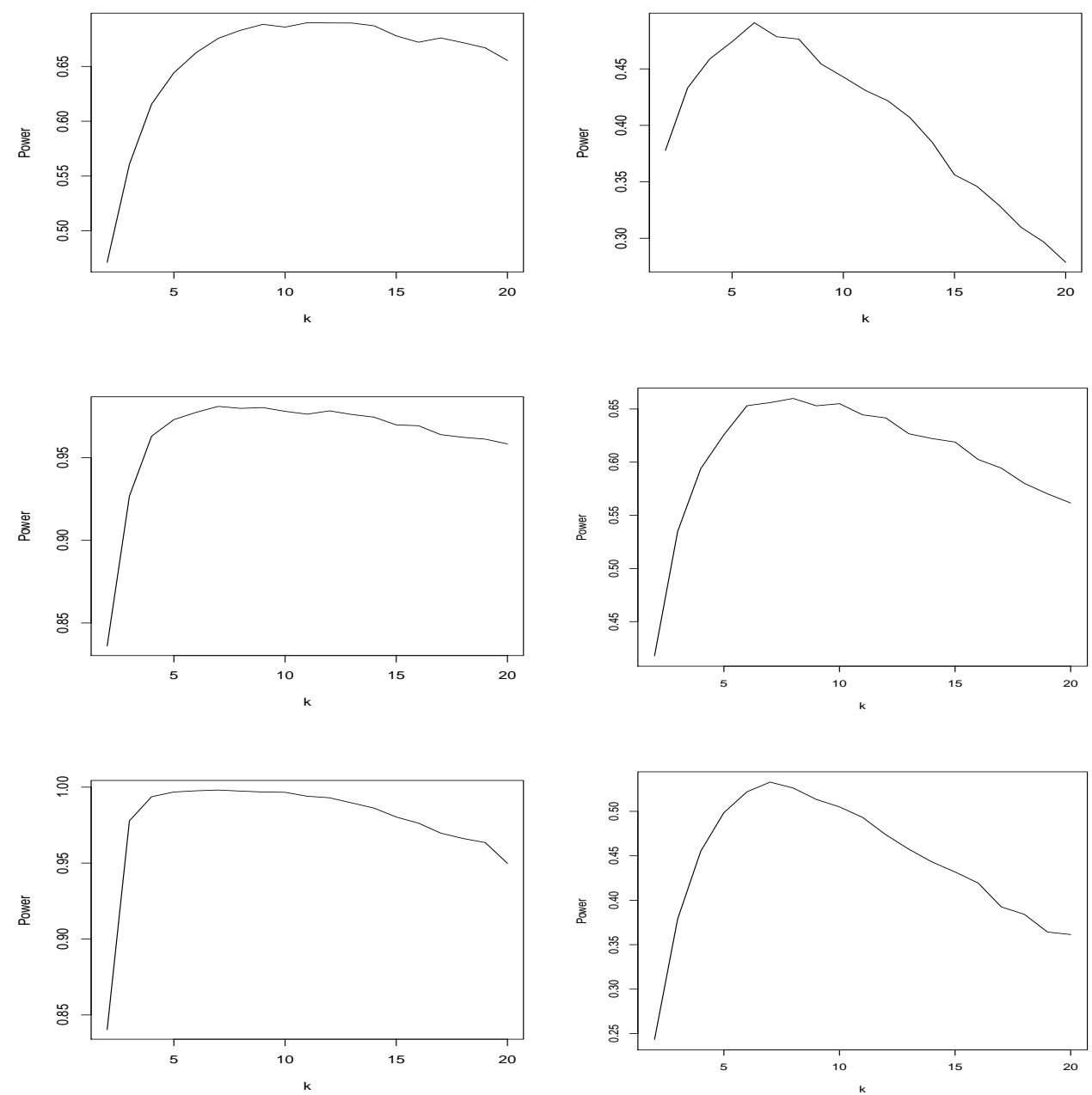

Figure 2: The power of the test of symmetry for the Clayton copula (left panels) and the Gumbel copula (right panels) for $\tau=0.25,0.5,0.75$ ( top to bottom ) and $1 \leq k \leq 20$. 


\subsection{Application to a Real Data Set}

In this subsection, we apply directional reflection asymmetric measures to analyze LifeCycleSavings data; see Belsley et al. (1980) for details. This data set has 50 observations on 5 variables. We use only three variables pob15 (percentage of population under 15), pop75 (percentage of the population over 75) and dpi (real per-capita disposable income).

The Spearman's rho, Kendall's tau and Pearson's moment correlation denoted by $r_{s}, t$ and $r$, respectively, are given in Table 4 . From the correlation values, we see that the variables pob75 and dpi have positive dependence and the variable pob15 has negative dependence on the variables pob75 and dpi. It is expected that if there is reflection asymmetry in the vector (pop15, po75, dpi), it must be in the direction $(1,-1,-1)$. The bivariate and trivariate scatter plots of real data and their uniform rank values are presented in Figure 3 . The estimated values of the asymmetry measures defined in (2.5) are given by $\widehat{\lambda}_{1}=0.001, \widehat{\lambda}_{2}=0.001, \widehat{\lambda}_{3}=-0.005$ and $\widehat{\lambda}_{4}=0.175$. Note that as defined in $(2.5), \lambda_{4}=\lambda^{(1,-1,-1)}$. Thus the maximum reflection asymmetry has occurred in the direction $(1,-1,-1)$, but the value of the test statistic 1.04 is less than the critical value 1.96, which is not significant at level $\alpha=0.05$.

For bivariate marginal variables $\widehat{\xi}_{1}\left(\right.$ pop 15, pop75) $=0, \widehat{\xi}_{1}($ pop $15, \mathrm{dpi})=-0.0024, \widehat{\xi}_{1}($ pop75, $\mathrm{dpi})=-0.018, \widehat{\xi}_{2}\left(\right.$ pop 15, po75) $=0.122, \widehat{\xi}_{2}($ pop $15, \mathrm{dpi})=0.226$ and $\widehat{\xi}_{2}($ pop $75, \mathrm{dpi})=0$. Note that as defined in (2.7), $\xi_{2}=\lambda^{(1,-1)}$. Thus the maximum reflection asymmetry for bivariate margins has occurred for the pair (pob15, dpi), which is in the direction $(1,-1)$. The value of the test statistic is 1.23 , which is not significant at the level $\alpha=0.05$. Thus there are no reflection asymmetry in bivariate cases for the pairs (pob15, pob75), (pob15, dpi) and (pob75, dpi).

We have also performed goodness of fit test to choose an appropriate copula for data. We fit the Normal, t-student, Frank, Clayton and Gumbel copulas. For the rotated vector (pop15, 1-po75, 1-dpi) the Frank copula with the parameter 7.78 is a good fit by using the Cramér-von Mises statistics (Genest et al. (2009)). We have also performed copula goodness of fit tests for bivariate marginal copulas. For the pairs (pob15, pob75) and (pob15, dpi) the Frank copula with the respective parameters -10.57 and -7.01 and for the pair (pop75, dpi) the Frank copula with the parameter 8.006 is the best fit.

Table 4: The Spearman's rho $\left(r_{s}\right)$, Kendall's tau $(t)$ and Pearson's moment correlation $(r)$ for three variables pop15, po75 and dpi of LifeCycleSavings data.

\begin{tabular}{cccccccccc}
\hline \hline Correlation & & $r_{s}$ & \multicolumn{3}{c}{$t$} & \multicolumn{3}{c}{$r$} \\
\hline \hline Variable & pop15 & pop75 & dpi & pop15 & pop75 & dpi & pop15 & pop75 & dpi \\
\hline \hline pop15 & 1.00 & -0.88 & -0.77 & 1.00 & -0.69 & -0.57 & 1.00 & -0.9 & -0.76 \\
pop75 & -0.88 & 1.00 & 0.80 & -0.69 & 1.00 & 0.60 & -0.90 & 1.00 & 0.78 \\
dpi & -0.77 & 0.80 & 1.00 & -0.57 & 0.60 & 1.00 & -0.76 & 0.78 & 1.00 \\
\hline
\end{tabular}



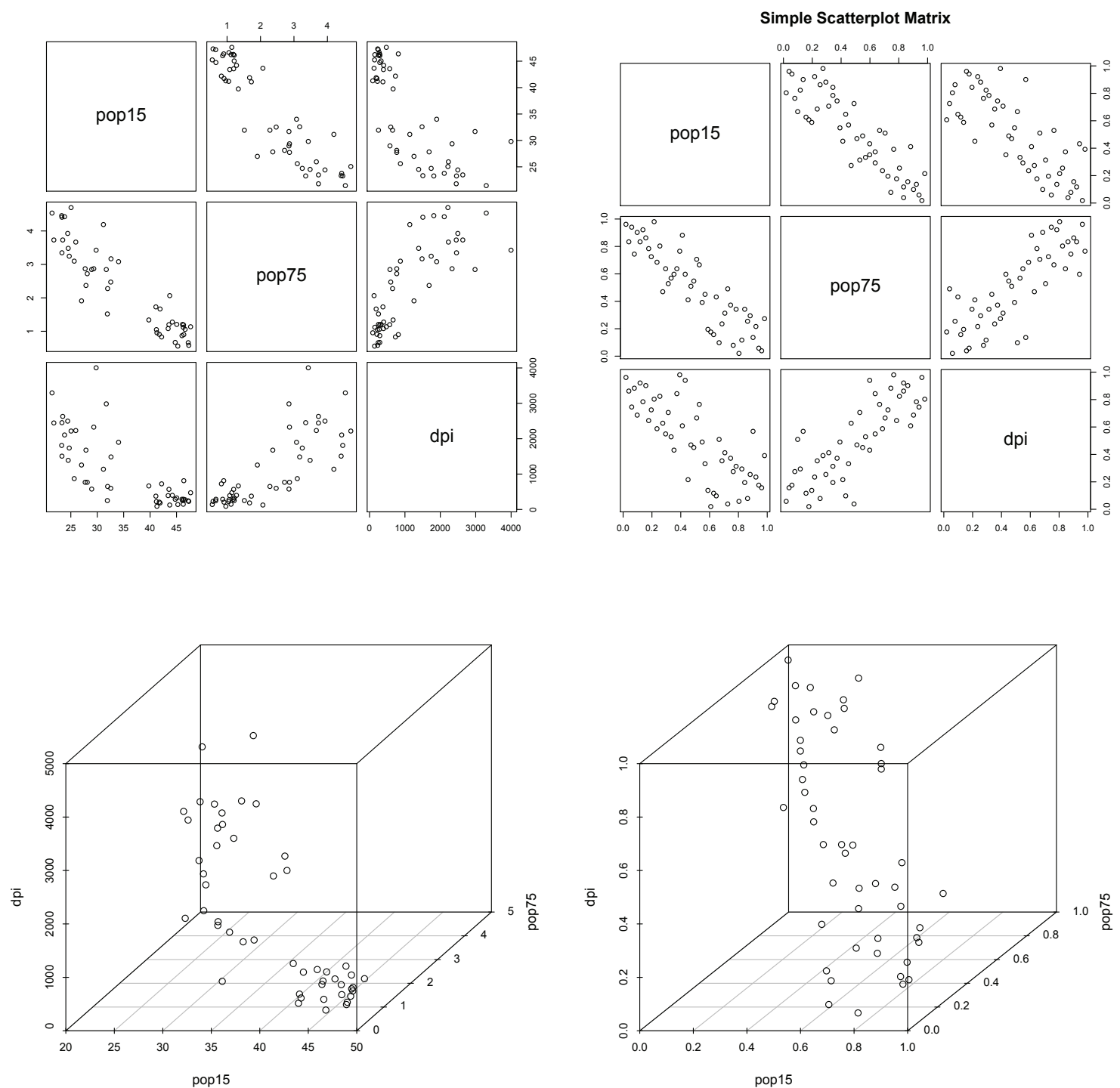

Figure 3: Two and three dimensional scatter plot of the variables pop15, pop75 and dpi in LifeCycleSavings data (the left panels) and their normalized ranks (the right panels). 


\section{Concluding Remarks}

A complementary to the goodness-of-fit methods for choosing a suitable copula is first testing the copula symmetry to suggest a sensible class of parametric families of copulas. Some tests for reflection asymmetry studied in the literature do not provide information about the direction of asymmetry. In this paper, we have introduced the concept of directional reflection asymmetry and proposed an index for measuring this kind of asymmetry in dimension $d>2$. The empirical estimate of the index is sample moments and could be readily estimated. A statistical test of asymmetry based on the proposed measure could be easily constructed using asymptotic normality of the corresponding estimator.

\section{Acknowledgements}

The authors wish to thank the two anonymous reviewers for their careful reading and insightful comments on an earlier version of this paper.

\section{References}

Alikhani-Vafa, A. and Dolati, A. (2018), A measure of radial asymmetry for bivariate copulas based on Sobolev norm. Hacettepe Journal of Mathematics and Statistics, 47(3), 649-658.

Belsley, D. A., Kuh. E., and Welsch, R. E. (1980), Regression Diagnostics. New York, Wiley.

Berg, D. (2009), Copula Goodness-Of-Fit Testing: An Overview and Power Comparison, The European Journal of Finance, 15, 675-701.

Bouzebda, S. and Cherfi, M. (2012), Test of symmetry based on copula function. Journal of Statistical Planning and Inference, 142, 1262-1271.

Cherubini, U., Mulinacci, S., Gobbi, F., and Romagnoli, S. (2011), Dynamic Copula methods in finance. John Wiley \& Sons.

Dehgani, A., Dolati, A., and Úbeda-Flores, M. (2013), Measures of radial asymmetry for bivariate random vectors. Statistical Papers, 54, 271-286.

Fang, K. T., Kotz, S., and Ng, K. W. (1989), Symmetric Multivariate and Related Distributions. Chapman \& Hall, London.

Fermanian, J. D., Radulovic, D., and Wegkamp, M. (2004), Weak convergence of empirical copula processes, Bernoulli, 10 (5), 847-860.

Genest, C., Remillard, B., and Beaudoin, D. (2009), Goodness-Of-Fit Tests for Copulas: A Review and a Power Study, Insurance: Mathematics and Economics, 44, 199-213.

Genest, C. and Neślehová, J. G. (2014), On tests of radial symmetry for bivariate copulas. Statistical Papers, 55, 1107-1119. 
Joe, H. (2015), Dependence Modeling with Copulas. CRC Press.

Krupskii, P. (2017), Copula-based measures of reflection and permutation asymmetry and statistical tests. Statistical Papers , 58(4), 1165-1187.

Modarres, R. (2017), Estimating the distribution function of symmetric pairs. Communications in Statistics-Theory and Methods, 46(4), 1843-1854.

Nelsen, R. B. (1993), Some concepts of bivariate symmetry. Journal of Nonparametric Statistics 3, 95-101.

Nelsen, R. B. (2006), An Introduction to Copulas. Second Edition. Springer, New York.

Radulovic, D., Wegkamp, M., and Zhao, Y. (2017), Weak convergence of empirical copula processes indexed by functions. Bernoulli, 23(4B), 3346-3384.

Rosco, J. and Joe, H. (2013), Measures of tail asymmetry for bivariate copulas. Statistical Papers, $54,709-726$.

Serfling, R. (2006), Multivariate Symmetry and Asymmetry. In Encyclopedia of Statistical Sciences, Second Edition (Kotz, S., Balakrishnan, N., Read, C. B. and Vidakovic, B., eds), Vol. 8, pp. 53385345. Wiley.

Sklar, A. (1959), Functions de répartition à $n$ dimensions et leurs marges. Publ. Inst. Statist. Univ. Paris 8, 229-231.

Trivedi, P. K. and Zimmer, D. M. (2007), Copula modeling: an introduction for practitioners. Foundations and Trends in Econometrics, 1(1), 1-111. 
